\title{
Treatment-resistant Delusional Dysmorphophobia
}

\author{
L Lavanya ${ }^{1}$, K Raman $^{2}$, Shanthi Nambi ${ }^{3}$, GS Chandraleka ${ }^{4}$
}

\begin{abstract}
Body dysmorphic disorder (BDD) is a relatively common disorder that consists of a distressing or impairing preoccupation with imagined or slight defects in appearance. It is important to recognize and appropriately treat BDD, as this disorder is associated with marked impairment in psychosocial functioning, notably poor quality of life and high suicidality rates. Herewith, we present a case of 44-year-old single unmarried male with chronic duration of preoccupation with imagined bodily defect with significant sociooccupational functioning. The patient was treated with multiple psychotropics, cognitive behavioral therapy, and a trial of electroconvulsive therapy (ECT). The response to treatment was almost negligible. The focus of discussion would be the presentation and treatment protocol followed in this resistant case.

Keywords: Delusional variant, Dysmorphophobia, Treatment resistance.

Indian Journal of Private Psychiatry (2019): 10.5005/jp-journals-10067-0035
\end{abstract}

\section{BACKGROUND}

Body dysmorphic disorder (BDD) is a relatively common, distressing, and disabling condition characterized by excessive concern about perceived defect in physical appearance that is either nonexistent or grossly exaggerated by the patients. ${ }^{1}$ Delusional beliefs and absence of insight are considered particularly incapacitating manifestations of this disorder. Treatment with selective serotonin reuptake inhibitor (SSRI), neuroleptics, and cognitive therapy are effective although a small number of case reports suggest that BDD may improve with electroconvulsive therapy (ECT). Here, we discuss a patient who was resistant to pharmacotherapy/psychotherapy and with ECT in various institutional settings.

\section{Case Description}

A 44-year-old single, unemployed, male from a rural area/ middle socioeconomic status was brought by his brother-in-law. Patient had given detailed history about the chronic course of his symptoms of change in his face that made him look very ugly, a belief that everybody was laughing at his facial features and therefore that he was object of ridicule. He believed that his face was transforming into that of a monkey.

Patient had avoidant behaviors like inability to go outside, going out only after sunset, and avoided meeting others mainly women in village. Chronic duration of preoccupation with imagined defect made him distressed, avoided social events with family and friends, difficult to concentrate on his job, and was contemplating suicide. He secluded himself from rest of society.

Patient developed anxiety in social situations due to his referential thinking. Patient visited multiple hospitals to treat his condition by selling the land and had frequent quarrels with his brother, which ended up in a physical fight over the property issues.

In addition, he had anhedonia, feeling of worthlessness, hopelessness, and suicidal ideation. He had depressive features but no biological dysfunction. No first rank symptom was evident. There was no history of substance abuse.

Patient was first among three children in the family, and his father died at the age of 35 and he committed suicide by hanging while being under the influence of alcohol. His grandmother had psychiatric illness and seizure disorder.

\footnotetext{
${ }^{1-4}$ Department of Psychiatry, Saveetha Medical College and Hospital, Chennai, Tamil Nadu, India
}

Corresponding Author: K Raman, Department of Psychiatry, Saveetha Medical College and Hospital, Chennai, Tamil Nadu, India, Phone: +91 9789039260, e-mail:dr_ramkrish@yahoo.com

How to cite this article: Lavanya L, Raman K, Nambi S, et al. Treatment-resistant Delusional Dysmorphophobia. Ind J Priv Psychiatry 2019;13(2):80-81.

Source of support: Nil

Conflict of interest: None

After many consultations, his belief that his face was deformed and looking like an animal was unshakeable. The belief was of delusional proportion. He had poor insight which lead to significant impairment in personal, social, and occupational domains.

Physical examination was normal. On mental state examination, the patient was alert, conscious, well-groomed, rapport was established, mood was subjectively worried, objectively sad, content of thought revealed dysmorphic delusion, and delusion of reference secondary to body dysmorphophobia. There was no perceptual abnormality. Insight was grade I. Routine hematological, biochemical tests, CT brain, and EEG were normal.

\section{Treatment History}

He was given adequate trial of fluoxetine up to $60 \mathrm{mg}$ and $30 \mathrm{mg}$ of buspirone for above symptoms. However, he showed limited response to this as well as to cognitive therapy. Later on, he was treated with low doses of amitriptyline and supportive therapy with which his symptoms persisted.

In 2002, he was started on olanzapine and received a maximum dose of $15 \mathrm{mg}$ beyond which he could not tolerate the drug. Since this did not reduce his symptoms, he was treated with trifluphenazine and risperidone. He was started on haloperidol and clonazepam when he had predominantly psychotic features and depressive features.

He was on haloperidol $10 \mathrm{mg}$, trihexyphenidyl $2 \mathrm{mg}$, clonazepam $0.25 \mathrm{mg}$, and sertraline $50 \mathrm{mg}$ for few months. Then he discontinued

() The Author(s). 2019 Open Access This article is distributed under the terms of the Creative Commons Attribution 4.0 International License (https://creativecommons. org/licenses/by-nc/4.0/), which permits unrestricted use, distribution, and non-commercial reproduction in any medium, provided you give appropriate credit to the original author(s) and the source, provide a link to the Creative Commons license, and indicate if changes were made. The Creative Commons Public Domain Dedication waiver (http://creativecommons.org/publicdomain/zero/1.0/) applies to the data made available in this article, unless otherwise stated. 
these drugs as he had side effects. Initially, he was treated with fluoxetine $40 \mathrm{mg}$, buspirone $10 \mathrm{mg}$, and two trials of ECT and continued the medications for few years.

He showed minimal improvement with fluoxetine $20 \mathrm{mg}$ but psychotic symptoms persisted. He was also tried on sertraline and clonazepam combination to maximum allowable dosage and for delusional component, and clozapine was started with 12.5 titrated up to $125 \mathrm{mg}$. Since there was no improvement, it was tapered and stopped. Later on, he was treated with pimozide $1 \mathrm{mg}$ for shorter duration.

Pharmacotherapy with cognitive behavior therapy (CBT) showed limited response for his condition. Behavioral experiments were used to reduce his social avoidance and mirror exposure to reduce his mirror avoidance. Cognitive behavior therapy techniques did not provide much improvement. Treatment was switched to meta-cognitive therapy (MCT) which focused on distress reduction. Patient is still on regular follow-up and there is little reduction in depressive features but referential delusional still persists.

\section{Discussion}

The patient described above was diagnosed to have treatmentresistant BDD based on patient's preoccupation with a nonexistent deformity which was of delusional intensity. Ensuing delusions of reference caused him to quit work and to shun social interactions. His anxious avoidance behavior was also of diagnostic importance. Chronic nature of illness, strong delusional belief with comorbid depression, and poor response to treatment led him to repeatedly seek treatment, but insight into illness was poor even after multiple trials of medications and psychoeducation. ${ }^{2,3}$

Selective serotonin reuptake inhibitors are the preferred choice of somatic treatments for BDD and have been reported to be effective for BDD-related delusions and comorbid depression with suicidal ideation. ECT was administered in our case to manage depression since he failed to respond to a variety of medication combinations that included SSRIs.

In many studies, it is advised to first treat BDD with SSRIs for 12-16 weeks, increasing medication to maximum allowable dosage, and to provide additional similarly prolonged trials with different SSRIs for initial treatment failures. Hence, our patient was considered to have failed the 4-month treatment with fluoxetine according to guidelines.

A range of SSRIs has been used in the treatment of BDD, including fluoxetine, sertraline, and paroxetine. Previous studies showed that most evidence for the efficacy of pharmacotherapies in BDD have shown response rates ranging from $53 \%$ to $70 \%$.

We intended to keep him under follow-up as the rapport with the psychologist was good. Behavioral experiments to address his impairment in socialization were the focus of treatment follow-up.

\section{Conclusion}

Selective serotonin reuptake inhibitors are generally the preferred class of somatic treatment, even in the presence of dysmorphic delusions. Clinical experience and guidelines suggest that SSRI augmentation with an atypical antipsychotic can be beneficial.
The assessment of the depressed patient referred for ECT should include careful screening for dysmorphic symptoms. ${ }^{6}$

Pharmacological and CBT-derived treatment options have proven valuable and efficacious in the treatment of BDD. BDDspecific CBT techniques have been found to be effective both in reducing symptom severity and in long-term management. This was followed in our study but still this case was unresponsive to therapy. ${ }^{7,8}$

Repetitive transcranial magnetic stimulation (rTMS) involves the use of focused electromagnetic fields to stimulate specific regions within the brain; the technique is currently being studied for use in depression and BDD.

Acceptance and commitment therapy (ACT) is a form of behavioral therapy based on the principle that patients consider internal processes aversive and thus can make ineffective attempts to change them and can be useful to assess the severity of BDD. ${ }^{9}$

\section{Clinical Significance}

The case described above stresses the need for early identification and initiation of therapy in BDD. The need for cognitive formulation and other biological methods of treatment in resistant cases was emphasized through the presentation. Resistant cases are associated with significant impairment and poor quality of life, hence the need for rapport building and improving insight.

\section{References}

1. Conroy M, Menard W, Fleming-Ives K, et al. Prevalence and clinical characteristics of body dysmorphic disorder in an adult inpatient setting. Gen Hosp Psychiatry 2008;30(1):67-72. DOI: 10.1016/ j.genhosppsych.2007.09.004.

2. Phillips KA, Menard W, Pagano ME, et al. Delusional versus nondelusional body dysmorphic disorder: clinical features and course of illness. J Psychiatr Res 2006;40(2):95-104. DOI: 10.1016/ j.jpsychires.2005.08.005.

3. Phillips KA, Menard W, Fay C, et al. Psychosocial functioning and quality of life in body dysmorphic disorder. Compr Psychiatry 2005;46(4):254-260. DOI: 10.1016/j.comppsych.2004.10.004.

4. Hollander E, Soorya L, Chaplin W, et al. A double-blind placebocontrolled trial of fluoxetine for repetitive behaviors and global severity in adult autism spectrum disorders. Am J Psychiatry 2012;169(3):292-929. DOI: 10.1176/appi.ajp.2011.10050764.

5. Phillips KA, Keshaviah A, Dougherty DD, et al. Pharmacotherapy relapse prevention in body dysmorphic disorder: a double-blind, placebo-controlled trial. Am J Psychiatry 2016;173(9):887-895. DOI: 10.1176/appi.ajp.2016.15091243.

6. Mahato RS, San Gabriel MC, Longshore CT, et al. A case of treatmentresistant depression and body dysmorphic disorder: the role of electroconvulsive therapy tevisited. Innov Clin Neurosci 2016; 13(7-8):37-40.

7. Veale D. Cognitive behavioral therapy for body dysmorphic disorder. Psychiatr Ann 2010;40(7):333-340. DOI: 10.3928/00485713-2010070106.

8. Krebs G, Fernández de la Cruz L, Mataix-Cols D. Recent advances in understanding and managing body dysmorphic disorder. Evid Based Ment Health 2017;20(3):71-75. DOI: 10.1136/eb-2017-102702.

9. Linde J, Rück C, Bjureberg J, et al. Acceptance-based exposure therapy for body dysmorphic disorder: a pilot study. Behav Ther 2015;46(4):423-431. DOI: 10.1016/j.beth.2015.05.002. 